\title{
Sandwich problems on orientations
}

\author{
Olivier Durand de Gevigney • Sulamita Klein • \\ Viet-Hang Nguyen · Zoltán Szigeti
}

Received: 1 February 2012 / Accepted: 3 February 2012 / Published online: 28 February 2012

(C) The Brazilian Computer Society 2012

\begin{abstract}
The graph sandwich problem for property $\Pi$ is defined as follows: Given two graphs $G_{1}=\left(V, E_{1}\right)$ and $G_{2}=\left(V, E_{2}\right)$ such that $E_{1} \subseteq E_{2}$, is there a graph $G=$ $(V, E)$ such that $E_{1} \subseteq E \subseteq E_{2}$ which satisfies property $\Pi$ ? We propose to study sandwich problems for properties $\Pi$ concerning orientations, such as Eulerian orientation of a mixed graph and orientation with given in-degrees of a graph. We present a characterization and a polynomial-time algorithm for solving the $m$-orientation sandwich problem.
\end{abstract}

Keywords Sandwich problems - Orientation of graphs . Submodular flows

\section{Introduction}

Given two graphs $G_{1}=\left(V, E_{1}\right)$ and $G_{2}=\left(V, E_{2}\right)$ with the same vertex set $V$ and $E_{1} \subseteq E_{2}$, a graph $G=(V, E)$ is called a sandwich graph for the pair $G_{1}, G_{2}$ if $E_{1} \subseteq E \subseteq E_{2}$. The graph sandwich problem for property $\Pi$ is defined as follows [12]:

O. Durand de Gevigney $(\bowtie) \cdot$ V.-H. Nguyen · Z. Szigeti Laboratoire G-SCOP, CNRS, Grenoble INP, UJF, 46, Avenue Félix Viallet, Grenoble 38000, France

e-mail: olivier.durand-de-gevigney@g-scop.inpg.fr

V.-H. Nguyen

e-mail: viet-hang.nguyen@g-scop.inpg.fr

Z. Szigeti

e-mail: Zoltan.Szigeti@g-scop.inpg.fr

S. Klein

Instituto de Matemática and COPPE, Universidade Federal

do Rio de Janeiro, Rio de Janeiro, Brazil

e-mail: sula@ cos.ufrj.br
Graph SANDWich Problem FOR Property $\Pi$ Instance: Given undirected graphs $G_{1}=\left(V, E_{1}\right)$ and $G_{2}=$ $\left(V, E_{2}\right)$ with $E_{1} \subseteq E_{2}$.

Question: Is there a graph $G=(V, E)$ such that $E_{1} \subseteq E \subseteq$ $E_{2}$ and $G$ satisfies property $\Pi$ ?

We call $E_{1}$ the mandatory edge set, $E_{0}=E_{2} \backslash E_{1}$ the optional edge set and $E_{3}$ the forbidden edge set, where $E_{3}$ denotes the set of edges of the complementary graph $\bar{G}_{2}$ of $G_{2}$. Thus any sandwich graph $G=(V, E)$ for the pair $G_{1}, G_{2}$ must contain all mandatory edges, no forbidden edges and may contain a subset of the optional edges. Graph sandwich problems have attracted much attention lately arising from many applications and as a natural generalization of recognition problems $[1-3,7,22,24]$. The recognition problem for a class of graphs $\mathcal{C}$ is equivalent to the graph sandwich problem in which $G_{1}=G_{2}=G$, where $G$ is the graph we want to recognize and property $\Pi$ is "to belong to class $\mathcal{C}$ ".

In this paper we propose to study sandwich problems for properties $\Pi$ concerning orientations, such as Eulerian orientation of a mixed graph and orientation with given indegrees of a graph, or more generally of a mixed graph.

The paper is organized as follows: Sect. 2 contains some basic definitions, notations and results. Section 3 contains some known results on degree constrained sandwich problems. We consider the undirected version and the directed version, the complexity, the characterization and the related optimization problems. We also define a simultaneous version and discuss its complexity. Section 4 focuses on Eulerian sandwich problems. We consider first undirected graphs and then directed graphs. These problems were already solved in [12], here we point out that the undirected case reduces to $T$-joins, while the directed case to circulations. We discuss the complexity of the problems and their characterizations and we also propose some mixed versions. 
In Sect. 5 we consider sandwich problems regarding an $m$ orientation, i.e., given undirected graphs $G_{1}=\left(V, E_{1}\right)$ and $G_{2}=\left(V, E_{2}\right)$ with $E_{1} \subseteq E_{2}$ and a non-negative integer vector $m$ on $V$, we show that it is polynomial to decide whether there exists a sandwich graph $G=(V, E)\left(E_{1} \subseteq E \subseteq E_{2}\right)$ that has an orientation $\vec{G}$ whose in-degree vector is $\vec{m}$ that is $d_{\vec{G}}^{-}(v)=m(v)$ for all $v \in V$. This result stands in contrast to the strongly connected $m$-orientation sandwich problem which we show is NP-complete. Section 6 is devoted to a new kind of sandwich problem where we may contract (and not delete) optional edges and property $\Pi$ is being bipartite.

\section{Definitions}

Undirected graphs Let $G=(V, E)$ be an undirected graph. For vertex sets $X$ and $Y$, the cut induced by $X$ is defined to be the set of edges of $G$ having exactly one endvertex in $X$ and is denoted by $\delta_{G}(X)$. The degree $\boldsymbol{d}_{G}(\boldsymbol{X})$ (or $d_{E}(X)$ ) of $X$ is the cardinality of the cut induced by $X$, that is, $d_{G}(X)=\left|\delta_{G}(X)\right|$. The number of edges between $X \backslash Y$ and $Y \backslash X$ is denoted by $\boldsymbol{d}_{\boldsymbol{G}}(\boldsymbol{X}, \boldsymbol{Y})$. The number of edges of $G$ having both (resp. at least one) endvertices in $X$ is denoted by $\boldsymbol{i}_{\boldsymbol{G}}(\boldsymbol{X})$ or $i_{E}(X)$ or simply $i(X)$ (resp. $\boldsymbol{e}_{\boldsymbol{G}}(\boldsymbol{X})$ ). It is well-known that (1) is satisfied for all $X, Y \subseteq V$,

$$
\begin{aligned}
d_{G}(X)+d_{G}(Y)= & d_{G}(X \cap Y)+d_{G}(X \cup Y) \\
& +2 d_{G}(X, Y) .
\end{aligned}
$$

We say that a vector $m$ on $V$ is the degree vector of $G$ if $d_{G}(v)=m(v)$ for all $v \in V$. For a vector $m$ on $V$, we consider $m$ as a modular function, that is, we use the notation $\boldsymbol{m}(\boldsymbol{X})=\sum_{v \in X} m(v)$. Let us recall that $d_{G}(X)$ is the degree function of $G$. We define $\hat{\boldsymbol{d}}_{\boldsymbol{G}}$ as the modular function defined by the degree vector $d_{G}(v)$ of $G$. Note that $\hat{d}_{G}(X)=d_{G}(X)+2 i_{G}(X) \forall X \subseteq V$.

We denote by $\boldsymbol{T}_{\boldsymbol{G}}$ the set of vertices of $G$ of odd degree. For an edge set $F$ of $G$, the subgraph induced by $F$, that is, $(V, F)$, is denoted by $\boldsymbol{G}(\boldsymbol{F})$. We say that $G$ is Eulerian if the degree of each vertex is even, that is, if $T_{G}=\emptyset$. Note that we do not suppose the graph to be connected.

Let $T$ be a vertex set in $G$. An edge set $F$ of $G$ is called $T$-join if the set of odd degree vertices in the subgraph induced by $F$ coincide with $T$, that is if $T_{G(F)}=T$. Given a cost vector on the edge set of $G$, a minimum cost T-join can be found in polynomial time by Edmonds and Johnson's algorithm [5].

Let $f$ be a non-negative integer vector on $V$. An edge set $F$ of $G$ is called an $f$-factor of $G$ if $f$ is the degree vector of $G(F)$, that is, $d_{F}(v)=f(v)$ for all $v \in V$. If $f(v)=1$ for all $v \in V$, then we say that $F$ is a 1 -factor or a perfect matching. An $f$-factor-if it exists_-can be found in polynomial time, see [20]. The graph $G$ is called 3-regular if each vertex is of degree 3. Note that for a 3-regular graph, the existence of two edge-disjoint perfect matchings is equivalent to the existence of three edge-disjoint perfect matchings which is equivalent to the 3-edge-colorability of the graph.

Directed graphs Let $D=(V, A)$ be a directed graph. For a vertex set $X$, the set of arcs of $D$ entering (resp. leaving) $X$ is denoted by $\varrho_{D}(\boldsymbol{X})\left(\operatorname{resp} . \boldsymbol{\delta}_{\boldsymbol{D}}(\boldsymbol{X})\right)$. The in-degree $\boldsymbol{d}_{\boldsymbol{D}}^{-}(\boldsymbol{X})$ (resp. out-degree $\boldsymbol{d}_{\boldsymbol{D}}^{+}(\boldsymbol{X})$ ) of $X$ is the number of arcs of $D$ entering (resp. leaving) $X$, that is $d_{D}^{-}(X)=\left|\varrho_{D}(X)\right|$ (resp. $\left.d_{D}^{+}(X)=\left|\delta_{D}(X)\right|\right)$. The set of arcs of $G$ having both endvertices in $X$ is denoted by $\boldsymbol{A}(\boldsymbol{X})$. The following equality will be used frequently without reference:

$d_{D}^{-}(X)-d_{D}^{+}(X)=\sum_{v \in X}\left(d_{D}^{-}(v)-d_{D}^{+}(v)\right)$.

We say that a vector $m$ on $V$ is the in-degree vector of $D$ if $d_{D}^{-}(v)=m(v)$ for all $v \in V$. Let us recall that $d_{D}^{-}(X)$ is the in-degree function of $D$. Let $f$ be a non-negative integer vector on $V$. An arc set $F$ of $D$ is called a directed $f$-factor of $D$ if $f$ is the in-degree vector of $D(F)$, that is, $d_{F}^{-}(v)=$ $f(v)$ for all $v \in V$.

We say that $D$ is Eulerian if the in-degree of $v$ is equal to the out-degree of $v$ for all $v \in V$, that is, $d_{D}^{-}(v)=d_{D}^{+}(v)$ for all $v \in V$. Note that we do not suppose the graph to be connected.

Let $f$ and $g$ be two vectors on the arcs of $D$ such that $f(e) \leq g(e)$ for all $e \in A$. A vector $x$ on the arcs of $D$ is a circulation if (3) and (4) are satisfied.

$x\left(\delta_{D}(v)\right)=x\left(\varrho_{D}(v)\right) \quad \forall v \in V$,

$f(e) \leq x(e) \leq g(e) \quad \forall e \in A$.

Note that if $f(e)=g(e)=1$ for all $e \in A$, then $D$ is Eulerian if and only if $f$ is a circulation. We will use the following characterization when a circulation exists.

Theorem 1 (Hoffmann [15]) Let $D=(V, A)$ be a directed graph and $f$ and $g$ two vectors on $A$ such that $f(e) \leq$ $g(e) \forall e \in A$. There exists a circulation in $D$ if and only if

$f\left(\varrho_{D}(X)\right) \leq g\left(\delta_{D}(X)\right) \quad \forall X \subseteq V$

We say that $H=(V, E \cup A)$ is a mixed graph if $E$ is an edge set and $A$ is an arc set on $V$. For an undirected graph $G=(V, E)$, if we replace each edge $u v$ by the arc $u v$ or $v u$, then we get the directed graph $\overrightarrow{\boldsymbol{G}}=(V, \vec{E})$. We say that $\vec{G}$ is an orientation of $G$.

Mixed graphs having Eulerian orientations are characterized as follows. 
Theorem 2 (Ford, Fulkerson [8]) A mixed graph $H=$ $(V, E \cup A)$ has an Eulerian orientation if and only if

$d_{A}^{-}(v)+d_{A}^{+}(v)+d_{E}(v) \quad$ is even $\forall v \in V$,

$d_{A}^{-}(X)-d_{A}^{+}(X) \leq d_{E}(X) \quad \forall X \subseteq V$.

The following theorem characterizes graphs having an orientation with a given in-degree vector.

Theorem 3 (Hakimi [13]) Given an undirected graph $G=$ $(V, E)$ and a non-negative integer vector $m$ on $V$, there exists an orientation $\vec{G}$ of $G$ whose in-degree vector is $m$ if and only if

$m(X) \geq i(X) \quad \forall X \subseteq V$,

$m(V)=|E|$.

Functions Let $b$ be a set function on the subsets of $V$. We say that $b$ is submodular if for all $X, Y \subseteq V$,

$$
b(X)+b(Y) \geq b(X \cap Y)+b(X \cup Y) .
$$

The function $b$ is called supermodular if $-b$ is submodular. A function is modular if it is supermodular and submodular. We will use frequently in this paper the following facts.

Claim 1 The degree function $d_{G}(Z)$ of an undirected graph $G$ and the in-degree function $d_{D}^{-}(Z)$ of a directed graph $D$ are submodular and the function $i(Z)$ is supermodular.

Theorem $4[17,21]$ The minimum value of a submodular function can be found in polynomial time.

Theorem 5 (Frank [9]) Let $b$ and $p$ be a submodular and a supermodular set function on $V$ such that $p(X) \leq b(X)$ for all $X \subseteq V$. Then there exists a modular function $m$ on $V$ such that $p(X) \leq m(X) \leq b(X)$ for all $X \subseteq V$. If $b$ and $p$ are integer valued then $m$ can also be chosen integer valued.

A pair $(p, b)$ of set functions on $2^{V}$ is a strong pair if $p$ (resp. $b$ ) is supermodular (submodular) and they are compliant, that is, for every pairwise disjoint triple $X, Y, Z$,

$b(X \cup Z)-p(Y \cup Z) \geq b(X)-p(Y)$.

Note that a pair $(\alpha, \beta)$ of modular functions is a strong pair if and only if $\alpha \leq \beta$. If $(p, b)$ is a strong pair then the polyhedron

$$
\begin{aligned}
Q(p, b)= & \left\{x \in \mathbb{R}^{V}: p(X) \leq x(X) \leq b(X),\right. \\
& \text { for every } X \subseteq V\}
\end{aligned}
$$

is called a generalized polymatroid (or a g-polymatroid). When $\alpha \leq \beta$ are modular, we also call the g-polymatroid $Q(\alpha, \beta)$ a box.
Theorem 6 (Frank, Tardos [11]) The intersection of an integral g-polymatroid $Q(p, b)$ and an integral box $Q(\alpha, \beta)$ is an integral g-polymatroid. It is nonempty if and only if $\alpha \leq b$ and $p \leq \beta$.

Matroids A set system $M=(V, \mathcal{F})$ is called a matroid if $\mathcal{F}$ satisfies the following three conditions:

(I1) $\emptyset \in \mathcal{F}$,

(I2) if $F \in \mathcal{F}$ and $F^{\prime} \subseteq F$, then $F^{\prime} \in \mathcal{F}$,

(I3) if $F, F^{\prime} \in \mathcal{F}$ and $|F|>\left|F^{\prime}\right|$, then there exists $f \in F \backslash$ $F^{\prime}$ such that $F^{\prime} \cup f \in \mathcal{F}$.

A subset $X$ of $V$ is called independent in $M$ if $X \in \mathcal{F}$, otherwise it is called dependent. The maximal independent sets of $V$ are the basis of $M$. Let $\mathcal{B}$ be the set of basis of $M$. Then $\mathcal{B}$ satisfies the following two conditions:

(B1) $\mathcal{B} \neq \emptyset$,

(B2) if $B, B^{\prime} \in \mathcal{B}$ and $b \in B \backslash B^{\prime}$, then there exists $b^{\prime} \in B^{\prime} \backslash$ $B$ such that $(B-b) \cup b^{\prime} \in \mathcal{B}$.

Conversely, if a set system $(V, \mathcal{B})$ satisfies $(B 1)$ and $(B 2)$, then $M=(V, \mathcal{F})$ is a matroid, where $\mathcal{F}=\{F \subseteq V$ : $\exists B \in \mathcal{B}, F \subseteq B\}$.

For $S \subset V$, the matroid $M \backslash S$ obtained from $M$ by deleting $S$ is defined as $M \backslash S=\left(V \backslash S,\left.\mathcal{F}\right|_{V \backslash S}\right)$, where $X \subseteq V \backslash S$ belongs to $\left.\mathcal{F}\right|_{V \backslash S}$ if and only if $X \in \mathcal{F}$. For $S \in \mathcal{F}$, the matroid $M / S$ obtained from $M$ by contracting $S$ is defined as $M / S=\left(V \backslash S, \mathcal{F}_{S}\right)$, where $X \subseteq V \backslash S$ belongs to $\mathcal{F}_{S}$ if and only if $X \cup S \in \mathcal{F}$. Let $\left\{V_{1}, \ldots, V_{l}\right\}$ be a partition of $V$ and $a_{1}, \ldots, a_{l}$ a set of non-negative integers. Then $M=(V, \mathcal{F})$ is a matroid, where $\mathcal{F}=\left\{F \subseteq V:\left|F \cap V_{i}\right| \leq a_{i}\right\}$, we call it partition matroid. The dual matroid $M^{*}$ of $M$ is defined as follows: the basis of $M^{*}$ are the complements of the basis of $M$.

Let $M=(V, \mathcal{F})$ be a matroid and $c$ a cost vector on $V=\left\{v_{1}, \ldots, v_{n}\right\}$. We can find a minimum cost basis $F_{n}$ of $M$ in polynomial time by the greedy algorithm: take a nondecreasing order of the elements of $V: c\left(v_{1}\right) \leq \cdots \leq c\left(v_{n}\right)$. Let $F_{0}$ be empty and for $i=1, \ldots, n$, let $F_{i}=F_{i-1}+v_{i}$ if $F_{i-1}+v_{i} \in \mathcal{F}$, otherwise let $F_{i}=F_{i-1}$.

If $M_{1}$ and $M_{2}$ are two matroids on the same ground set $V$, then we can find a common basis of $M_{1}$ and $M_{2}$ in polynomial time (if there exists one) by the matroid intersection algorithm of Edmonds [4].

Theorem 7 (Edmonds, Rota [18]) For an integer-valued, non-decreasing, submodular function $b$ defined on a ground set $S$, the set $\left\{F \subseteq S ;\left|F^{\prime}\right| \leq b\left(F^{\prime}\right)\right.$ for all $\left.\emptyset \neq F^{\prime} \subseteq F\right\}$ forms the set of independent sets of a matroid $M_{b}$ whose rank function $r_{b}$ is given by

$r_{b}(Z)=\min \{b(X)+|Z-X|, X \subseteq Z\}$. 
Given an undirected graph $G=(V, E)$ and a nonnegative integer vector $m$ on $V$, let $\bar{m}^{G}=\bar{m}$ be the set function defined on $E$ by $\bar{m}(F)=m(V(F))$ where $V(F)$ is the set of vertices covered by $F$. One can easily check that $\bar{m}$ is integer valued, non-decreasing and submodular. Thus, by Theorem $7, \bar{m}$ defines a matroid $M_{\bar{m}}$. The following claim is straightforward.

Claim 2 The set $\left\{F \subseteq E: m(X) \geq i_{F}(X), \forall X \subseteq V\right\}$ is the set of independent sets of the matroid $M_{\bar{m}}$.

\section{Degree constrained sandwich problems}

Before studying sandwich problems on orientations of given in-degrees, let us start as a warming up by considering sandwich problems for undirected and directed graphs of given degrees. These problems reduce to the undirected and directed $f$-factor problems. We mention that the directed case is much easier than the undirected case because the addition of an arc in a directed graph contributes only to the in-degree of the head and not of the tail, while the addition of an edge in an undirected graph contributes to the degree of both endvertices. This section contains no new results, we added it for the sake of completeness.

\subsection{Undirected graphs}

\section{Undirected Degree Constrained SANDWICH PROB-} LEM

Instance: Given undirected graphs $G_{1}=\left(V, E_{1}\right)$ and $G_{2}=$ ( $\left.V, E_{2}\right)$ with $E_{1} \subseteq E_{2}$ and a non-negative integer vector $f$ on $V$.

Question: Does there exist a sandwich graph $G=(V, E)$ $\left(E_{1} \subseteq E \subseteq E_{2}\right)$ such that $d_{G}(v)=f(v)$ for all $v \in V$ ?

Complexity: It is in P because the answer is YES if and only if there exists an $\left(f(v)-d_{G_{1}}(v)\right)$-factor in the optional graph $G_{0}=\left(V, E_{0}\right)$.

Characterization: The general $f$-factor theorem due to Tutte [25] can be applied to get a characterization.

Optimization: The minimum cost $f$-factor problem in undirected graphs can be solved in polynomial time, see Schrijver [20].

\section{Simultaneous Undirected Degree Constrained} SANDWICH PROBLEM

Instance: Given two edge-disjoint graphs $G_{1}=\left(V, E_{1}\right)$ and $G_{2}=\left(V, E_{2}\right)$ in $G_{3}=\left(V, E_{3}\right)$ and two non-negative integer vectors $f_{1}$ and $f_{2}$ on $V$.

Question: Do there exist simultaneously sandwich graphs $\hat{G}_{1}=\left(V, \hat{E}_{1}\right)\left(E_{1} \subseteq \hat{E}_{1} \subseteq E_{3}\right)$ and $\hat{G}_{2}=\left(V, \hat{E}_{2}\right)\left(E_{2} \subseteq\right.$
$\left.\hat{E}_{2} \subseteq E_{3}\right)$ such that $\hat{E}_{1} \cap \hat{E}_{2}=\emptyset$ and $d_{\hat{G}_{1}}(v)=f_{1}(v)$ and $d_{\hat{G}_{2}}(v)=f_{2}(v)$ for all $v \in V$ ?

Complexity: It is NP-complete because it contains as a special case whether there exist two edge-disjoint perfect matchings so 3-edge-colorability of 3-regular graphs. Indeed, let $G=(V, E)$ be an arbitrary 3-regular graph. Let $G_{1}$ and $G_{2}$ be the edgeless graph on $V, G_{3}=G$ and $f_{1}(v)=$ $f_{2}(v)=1$ for all $v \in V$. Then the sandwich graphs $\hat{G}_{1}$ and $\hat{G}_{2}$ exist if and only if $\hat{E}_{1}$ and $\hat{E}_{2}$ are edge-disjoint perfect matchings of $G$ or equivalently, if there exists a 3-edgecoloring of $G$. Since the problem of 3-edge-colorability of 3-regular graphs is NP-complete [16], so is our problem.

\subsection{Directed graphs}

\section{Directed Degree Constrained SAndwich Prob-} LEM

Instance: Given directed graphs $D_{1}=\left(V, A_{1}\right)$ and $D_{2}=$ $\left(V, A_{2}\right)$ with $A_{1} \subseteq A_{2}$ and a non-negative integer vector $f$ on $V$.

Question: Does there exist a sandwich graph $D=(V, A)$ $\left(A_{1} \subseteq A \subseteq A_{2}\right)$ such that $d_{D}^{-}(v)=f(v)$ for all $v \in V$ ?

Complexity + Characterization: It is in P because the answer is YES if and only if there exists a directed $(f(v)-$ $\left.d_{D_{1}}^{-}(v)\right)$-factor in the optional directed graph $D_{0}=\left(V, A_{0}\right)$, hence we have the following.

Theorem 8 The Directed Degree Constrained SANDWICH PROBLEM has a YES answer if and only if $d_{D_{2}}^{-}(v) \geq f(v) \geq d_{D_{1}}^{-}(v)$ for all $v \in V$.

Optimization: The feasible arc sets form the basis of a partition matroid, so the greedy algorithm provides a minimum cost solution.

\section{Simultaneous Directed Degree Constrained} SANDWICH PROBLEM 1

Instance: Given two arc-disjoint directed graphs $D_{1}=$ $\left(V, A_{1}\right)$ and $D_{2}=\left(V, A_{2}\right)$ in $D_{3}=\left(V, A_{3}\right)$ and two nonnegative integer vectors $f_{1}$ and $f_{2}$ on $V$.

Question: Do there exist simultaneously sandwich graphs $\hat{D}_{1}=\left(V, \hat{A}_{1}\right)\left(A_{1} \subseteq \hat{A}_{1} \subseteq A_{3}\right)$ and $\hat{D}_{2}=\left(V, \hat{A}_{2}\right)\left(A_{2} \subseteq\right.$ $\left.\hat{A}_{2} \subseteq A_{3}\right)$ such that $\hat{A}_{1} \cap \hat{A}_{2}=\emptyset$ and $d_{\hat{D}_{1}}(v)=f_{1}(v)$ and $d_{\hat{D}_{2}}^{-}(v)=f_{2}(v)$ for all $v \in V$ ?

Complexity: It is in P because the answer is YES if and only if $d_{D_{3}}^{-}(v) \geq f_{1}(v)+f_{2}(v), f_{1}(v) \geq d_{D_{1}}^{-}(v)$ and $f_{2}(v) \geq$ $d_{D_{2}}^{-}(v)$ for all $v \in V$.

Simultaneous Directed Degree Constrained SANDWICH PROBLEM 2 
Instance: Given directed graphs $D_{1}=\left(V, A_{1}\right)$ and $D_{2}=$ ( $V, A_{2}$ ) with $A_{1} \subseteq A_{2}$ and two non-negative integer vectors $f$ and $g$ on $V$.

Question: Does there exist a sandwich graph $D=(V, A)$ $\left(A_{1} \subseteq A \subseteq A_{2}\right)$ such that $d_{D}^{-}(v)=f(v)$ and $d_{D}^{+}(v)=g(v)$ for all $v \in V$.

Complexity: The feasible arc sets for the in-degree constraint form the basis of a partition matroid and the feasible arc sets for the out-degree constraint form the basis of a partition matroid. The answer is YES if and only if there exists a common basis in these two matroids. Thus it is in $\mathrm{P}$ by the matroid intersection algorithm of Edmonds [4].

\section{Eulerian sandwich problems}

In this section we consider first two problems that were already solved in [12]: Eulerian sandwich problems for undirected and directed graphs. We point out that the undirected case reduces to $T$-joins, while the directed case to circulations. We show that in both cases the simultaneous versions are NP-complete.

Then we propose to study the problem in mixed graphs. We show two cases that can be solved. The first case will be solved by the Discrete Separation Theorem 5 of Frank [9], while the second case reduces to the DIRECTED EULERIAN SANDWICH PROBLEM. The general case, however, remains open.

\subsection{Undirected graphs}

\section{UndiReCTEd EulERIAN SANDWiCH PROBlem}

Instance: Given undirected graphs $G_{1}=\left(V, E_{1}\right)$ and $G_{2}=$ $\left(V, E_{2}\right)$ with $E_{1} \subseteq E_{2}$.

Question: Does there exist a sandwich graph $G=(V, E)$ $\left(E_{1} \subseteq E \subseteq E_{2}\right)$ that is Eulerian?

Complexity: It is in P because the answer is YES if and only if there exists a $T_{G_{1}}$-join in the optional graph $G_{0}$.

Characterization: The answer is YES if and only if each connected component of $G_{0}$ contains an even number of vertices of $T_{G_{1}}$.

Optimization: The minimum cost $T$-join problem can be solved in polynomial time [5].

\section{Simultaneous Undirected Eulerian Sandwich} PROBLEM

Instance: Given two edge-disjoint graphs $G_{1}=\left(V, E_{1}\right)$ and $G_{2}=\left(V, E_{2}\right)$ in $G_{3}=\left(V, E_{3}\right)$.

Question: Do there exist simultaneously Eulerian sandwich graphs $\hat{G}_{1}=\left(V, \hat{E}_{1}\right)\left(E_{1} \subseteq \hat{E}_{1} \subseteq E_{3}\right)$ and $\hat{G}_{2}=\left(V, \hat{E}_{2}\right)$ $\left(E_{2} \subseteq \hat{E}_{2} \subseteq E_{3}\right)$ such that $\hat{E}_{1} \cap \hat{E}_{2}=\emptyset$ ?
Complexity: It is NP-complete because it contains as a special case whether there exist two edge-disjoint perfect matchings so 3-colorability of 3-regular graphs. Indeed, let $G=(V, E)$ be an arbitrary 3-regular graph. Let $G_{3}$ be obtained from $G$ by adding 2 edge-disjoint perfect matchings $M_{1}$ and $M_{2}$ to $G$, let $G_{1}=\left(V, M_{1}\right)$ and $G_{2}=\left(V, M_{2}\right)$. Then the Eulerian sandwich graphs $\hat{G}_{1}$ and $\hat{G}_{2}$ exist if and only if $\hat{E}_{1} \backslash M_{1}$ and $\hat{E}_{2} \backslash M_{2}$ are edge-disjoint perfect matchings of $G$ or equivalently, if there exists a 3-edge-coloring of $G$. Since the problem of 3-edge-colorability of 3-regular graphs is NP-complete [16], so is our problem.

\subsection{Directed graphs}

\section{Directed Eulerian SANDwich Problem}

Instance: Given directed graphs $D_{1}=\left(V, A_{1}\right)$ and $D_{2}=$ $\left(V, A_{2}\right)$ with $A_{1} \subseteq A_{2}$.

Question: Does there exist a sandwich graph $D=(V, A)$ $\left(A_{1} \subseteq A \subseteq A_{2}\right)$ that is Eulerian?

Complexity: It is in $\mathrm{P}$ because it can be reformulated as a circulation problem: let $f(e)=1, g(e)=1$ if $e \in A_{1}$ and $f(e)=0, g(e)=1$ if $e \in A_{0}$. This way the arcs of $A_{1}$ are forced and the arcs of $A_{0}$ can be chosen if necessary.

Characterization: The answer is YES if and only if $d_{D_{1}}^{-}(X) \leq d_{D_{2}}^{+}(X)$ for all $X \subseteq V$ by Theorem 1.

Optimization: The minimum cost circulation problem can be solved in polynomial time, see Tardos [23].

\section{Simultaneous Directed Eulerian SANDwich PROBLEM}

Instance: Given two arc-disjoint directed graphs $D_{1}=$ $\left(V, A_{1}\right)$ and $D_{2}=\left(V, A_{2}\right)$ in $D_{3}=\left(V, A_{3}\right)$.

Question: Do there exist simultaneously Eulerian sandwich graphs $\hat{D}_{1}=\left(V, \hat{A}_{1}\right)\left(A_{1} \subseteq \hat{A}_{1} \subseteq A_{3}\right)$ and $\hat{D}_{2}=\left(V, \hat{A}_{2}\right)$ $\left(A_{2} \subseteq \hat{A}_{2} \subseteq A_{3}\right)$ such that $\hat{A}_{1} \cap \hat{A}_{2}=\emptyset$ ?

Complexity: It is NP-complete, it contains as a special case $\left(D_{1}=\left(V, t_{1} s_{1}\right), D_{2}=\left(V, t_{2} s_{2}\right)\right.$ and $\left.D_{3}=D\right)$ the following directed 2-commodity integral flow problem that is NPcomplete [6]: Given a directed graph $D$ and two pairs of vertices, $s_{1}, t_{1}$ and $s_{2}, t_{2}$, decide whether there exist a path from $s_{1}$ to $t_{1}$ and a path from $s_{2}$ to $t_{2}$ that are arc-disjoint.

\subsection{Mixed graphs}

\section{Mixed Eulerian SAndwich Problem}

Instance: Given mixed graphs $H_{1}=\left(V, E_{1} \cup A_{1}\right)$ and $H_{2}=$ $\left(V, E_{2} \cup A_{2}\right)$ with $E_{1} \subseteq E_{2}, A_{1} \subseteq A_{2}$.

Question: Does there exist a sandwich mixed graph $H=$ $(V, E \cup A)\left(E_{1} \subseteq E \subseteq E_{2}, A_{1} \subseteq A \subseteq A_{2}\right)$ that has an Eulerian orientation? 
Complexity: We provide two special cases that can be treated, while the general problem remains open.

SPecial Case 1: $E_{1}=E_{2}=E$ and $d_{A_{2}}^{+}(X)-d_{A_{1}}^{-}(X)+$ $\hat{d}_{E}(X)$ is even for all $X \subseteq V$.

Characterization + Complexity: We show that the problem is in $\mathrm{P}$ and we provide a characterization.

Theorem 9 The MiXed Eulerian SANDwich Problem with $E_{1}=E_{2}=E$ and $d_{A_{2}}^{+}(X)-d_{A_{1}}^{-}(X)+\hat{d}_{E}(X)$ is even for all $X \subseteq V$ has $a$ YES answer if and only if

$d_{A_{1}}^{-}(X)-d_{A_{2}}^{+}(X) \leq d_{E}(X) \forall X \subseteq V$.

In particular, this problem is in $\mathrm{P}$.

Proof By the result of Sect. 4.2, the answer is YES if and only if there exists an orientation $\vec{E}$ of $E$ such that, $\forall X \subseteq V$, $d_{A_{1} \cup \vec{E}}^{-}(X) \leq d_{A_{2} \cup \vec{E}}^{+}(X)$ or equivalently

$d_{\vec{E}}^{-}(X)-d_{\vec{E}}^{+}(X) \leq d_{A_{2}}^{+}(X)-d_{A_{1}}^{-}(X)$.

Let $m$ be the in-degree vector of $\vec{E}$. Then $d_{\vec{E}}^{-}(X)-d_{\vec{E}}^{+}(X)=$ $\sum_{v \in X}\left(d_{\vec{E}}^{-}(v)-d_{\vec{E}}^{+}(v)\right)=\sum_{v \in X}\left(2 d_{\vec{E}}^{-}(v)-d_{E}(v)\right)=$ $2 m(X)-\hat{d}_{E}(X)$, and (12) becomes

$2 m(X) \leq d_{A_{2}}^{+}(X)-d_{A_{1}}^{-}(X)+\hat{d}_{E}(X)$.

Let $b(X)=\frac{1}{2}\left(d_{A_{2}}^{+}(X)-d_{A_{1}}^{-}(X)+\hat{d}_{E}(X)\right)$. Then $b$, being the sum of a modular function and a submodular function $\left(b(X)=\frac{1}{2} \sum_{v \in X}\left(d_{A_{1}}^{+}(v)-d_{A_{1}}^{-}(v)+d_{E}(v)\right)+d_{A_{0}}^{+}(X)\right)$, is a submodular function and, by the assumption, it is integer valued. By Theorem 3, an orientation $\vec{E}$ satisfying (12) exists if and only if there exists a vector $m$ such that $i_{E}(X) \leq m(X) \leq b(X)$, that is, by Claim 1 and Theorem 5 , if and only if $i_{E}(X) \leq b(X)$. This is equivalent to (11) and can be decided in polynomial time by Theorem 4 , namely the submodular function $b^{\prime}(X)=b(X)-i_{E}(X)$ must have minimum value 0 .

\section{Special Case 2: $E_{1}=\emptyset$.}

Characterization + Complexity: It is in P because it can be reformulated as the following problem: We create two copies of each edge in $E_{2}$ and orient them in opposite directions. Denote this arc set by $\overrightarrow{E_{2}^{2}}$. It is not difficult to see that the graph $\left(V, E_{2} \cup A_{2}\right)$ has a subgraph containing $\left(V, A_{1}\right)$ with an Eulerian orientation if and only if the graph $\left(V, \overrightarrow{E_{2}^{2}} \cup A_{2}\right)$ has a directed Eulerian subgraph containing $\left(V, A_{1}\right)$. Indeed, in such a graph, if every edge of $E_{2}$ is used at most once, we are done. If some edge of $E_{2}$ is used twice, as two arcs in opposite directions, we can just remove these two arcs, the obtained graph remaining Eulerian and containing $\left(V, A_{1}\right)$. Now applying the result for DIRECTED EULERIAN SANDWICH PROBLEM we have

Theorem 10 The Mixed Eulerian Sandwich ProbLEM with $E_{1}=\emptyset$ has a YES answer if and only if

$d_{A_{1}}^{-}(X)-d_{A_{2}}^{+}(X) \leq d_{E_{2}}(X) \forall X \subseteq V$.

In particular, this problem is in $\mathrm{P}$.

Proof Let $D_{1}=\left(V, A_{1}\right)$ and $D_{2}=\left(V, A_{2} \cup \overrightarrow{E_{2}^{2}}\right)$. By the arguments above, the Mixed Eulerian SANDWich ProbLEM with $E_{1}=\emptyset$ has a solution if and only if there is an Eulerian sandwich graph for $D_{1}$ and $D_{2}$ or equivalently, $d_{D_{1}}^{-}(X) \leq d_{D_{2}}^{+}(X)$ for all $X \subseteq V$. By $d_{D_{2}}^{+}(X)=d_{A_{2}}^{+}(X)+$ $d_{E_{2}}(X)$, we have $d_{A_{1}}^{-}(X)-d_{A_{2}}^{+}(X) \leq d_{E_{2}}(X)$ for all $X \subseteq V$. Note that $d_{E_{2}}(X)+d_{A_{2}}^{+}(X)-d_{A_{1}}^{-}(X)$ is a submodular function, and hence by Theorem 4, (14) can be verified in polynomial time.

\section{$5 m$-orientation sandwich problems}

In this section we consider the sandwich problem where the property $\Pi$ is to have an orientation of given in-degrees.

\section{$5.1 m$-Orientation}

$m$-ORIENTATION SANDWICH PROBLEM

Instance: Given undirected graphs $G_{1}=\left(V, E_{1}\right)$ and $G_{2}=$ $\left(V, E_{2}\right)$ with $E_{1} \subseteq E_{2}$ and a non-negative integer vector $m$ on $V$.

Question: Does there exist a sandwich graph $G=(V, E)$ $\left(E_{1} \subseteq E \subseteq E_{2}\right)$ that has an orientation $\vec{G}$ whose in-degree vector is $m$ that is $d_{\vec{G}}^{-}(v)=m(v)$ for all $v \in V$ ?

Characterization: We prove the following theorem.

Theorem 11 The following assertions are equivalent.

(a) The $m$-Orientation SAndwich Problem has a YES answer.

(b) $E_{1}$ is independent in $M_{\bar{m}}$ and $M_{\bar{m}}$ has an independent set of size $m(V)$.

(c) $r_{\bar{m}}\left(E_{1}\right)=\left|E_{1}\right|$ and $r_{\bar{m}}\left(E_{2}\right) \geq m(V)$.

(d) $i_{E_{1}}(X) \leq m(X) \leq e_{E_{2}}(X)$ for all $X \subseteq V$.

\section{Proof}

(a) Implies (d). Let $X \subseteq V$. Since each edge of $G_{1}$ in $X$ contributes 1 to $m(X)$, we have $i_{E_{1}}(X) \leq m(X)$. On the other hand, the edges of $G_{2}$ that have no end-vertex in $X$ cannot contribute 1 to $m(X)$, so we have $m(X) \leq e_{E_{2}}(X)$. 
(d) Implies (c). Let $F$ be a subset of $E_{1}$ and $X=V(F)$. The condition $i_{E_{1}}(X) \leq m(X)$ implies $|F| \leq m(V(F))=$ $\bar{m}(F)$, that is, $\left|E_{1}\right| \leq \bar{m}(F)+\left|E_{1} \backslash F\right|$. By Theorem 7, $r_{\bar{m}}\left(E_{1}\right) \geq\left|E_{1}\right|$, or equivalently $r_{\bar{m}}\left(E_{1}\right)=\left|E_{1}\right|$. Let now $F$ be a subset of $E_{2}$ and $X=V \backslash V(F)$. The condition $m(X) \leq$ $e_{E_{2}}(X)$ implies that $m(V) \leq m(V(F))+e_{E_{2}}(V-V(F)) \leq$ $\bar{m}(F)+\left|E_{2} \backslash F\right|$. By Theorem 7, $r_{\bar{m}}\left(E_{2}\right) \geq m(V)$.

(c) Implies (b). By definition.

(b) Implies (a). By (b), $E_{1}$ is independent in $M_{\bar{m}}$ and there exists an independent in $M_{\bar{m}}$ of size $m(V)$. Therefore, by (I3), there exists an independent set $E$ of size $m(V)$ that contains $E_{1}$. By Theorem 3 and Claim 2, $E$ is a solution of the $m$-ORIENTATION SANDWICH PROBLEM.

We say that a subset $F$ of $E_{0}$ is feasible if ( $\left.V, F \cup E_{1}\right)$ has an $m$-orientation. The next corollary of Theorem 11 characterizes the feasible sets.

Corollary 1 If the $m$-ORIENTATION SANDWICH PROBLEM has a YES answer, then a subset $F$ of $E_{0}$ is feasible if and only if $F$ is a base of the matroid $M_{\bar{m}} / E_{1}$.

Complexity: The condition (d) of Theorem 11 can be verified in polynomial time by Theorem 4, so the $m$-Orientation SANDWICH PROBlem is in P.

Optimization: The minimum cost version of the problem can be solved in polynomial time. First, we find an optimal feasible subset $F$ by greedy algorithm. Then we can orient the edges of $F \cup E_{1}$ using a known algorithm. (See [10] for example.)

Corollary 1 and the matroid intersection algorithm of Edmonds [4] imply that the two following simultaneous versions of the $m$-ORIENTATION SANDWICH PROBLEM are also in $\mathrm{P}$.

\section{Simultaneous $m$-ORIENTATION SANDWich PROBlem 1} Instance: Given two edge-disjoint undirected subgraphs $G_{1}=\left(V, E_{1}\right)$ and $G_{2}=\left(V, E_{2}\right)$ of an undirected graph $G_{3}=\left(V, E_{3}\right)$ and two non-negative integer vectors $m_{1}$ and $m_{2}$ on $V$.

Question: Do there exist simultaneously edge-disjoint sandwich graphs $\hat{G}_{1}=\left(V, \hat{E}_{1}\right)\left(E_{1} \subseteq \hat{E}_{1} \subseteq E_{3}\right)$ and $\hat{G}_{2}=$ $\left(V, \hat{E}_{2}\right)\left(E_{2} \subseteq \hat{E}_{2} \subseteq E_{3}\right)$ such that $\hat{G}_{i}$ has an orientation whose in-degree vector is $m_{i}$ for $i \in\{1,2\}$ ?

Note that the two input matroids for the matroid intersection algorithm must be taken as $\left(M_{\bar{m}_{1}}^{G_{1}} / E_{1}\right) \backslash E_{2}$ and the dual matroid of $\left(M_{\bar{m}_{2}}^{G_{2}} / E_{2}\right) \backslash E_{1}$.

Simultaneous $m$-ORIENTATION SANDWiCH PROBlem 2 Instance: Given two undirected subgraphs $G_{1}=\left(V, E_{1}\right)$ and $G_{2}=\left(V, E_{2}\right)$ of an undirected graph $G_{3}=\left(V, E_{3}\right)$ and two non-negative integer vectors $m_{1}$ and $m_{2}$ on $V$.

Question: Does there exist an edge set $F$ in $E_{3} \backslash\left(E_{1} \cup E_{2}\right)$ such that the graph $G_{i}=\left(V, E_{i} \cup F\right)$ admits an orientation whose in-degree vector is $m_{i}$ for $i \in\{1,2\}$ ?

\subsection{Strongly connected $m$-orientation}

\section{Strongly Connected $m$-Orientation SANDWich} PROBLEM

Instance: Given undirected graphs $G_{1}=\left(V, E_{1}\right)$ and $G_{2}=$ $\left(V, E_{2}\right)$ with $E_{1} \subseteq E_{2}$ and a non-negative integer vector $m$ on $V$.

Question: Does there exist a sandwich graph $G=(V, E)$ $\left(E_{1} \subseteq E \subseteq E_{2}\right)$ that has a strongly connected orientation $\vec{G}$ whose in-degree function is $m$ ?

Complexity: It is NP-complete because the special case $E_{1}=\emptyset, m(v)=1 \forall v \in V$ is equivalent to decide if $G_{2}$ has a Hamiltonian cycle.

\section{$5.3\left(m_{1}, m_{2}\right)$-orientation}

$\left(m_{1}, m_{2}\right)$-ORIEnTATION SANDWich PROBlem

Instance: Given undirected graphs $G_{1}=\left(V, E_{1}\right)$ and $G_{2}=$ $\left(V, E_{2}\right)$ with $E_{1} \subseteq E_{2}$ and non-negative integer vectors $m_{1}$ and $m_{2}$ on $V$.

Question: Does there exist a sandwich graph $G=(V, E)$ ( $E_{1} \subseteq E \subseteq E_{2}$ ) that has an orientation $\vec{G}$ whose in-degree vector is $m_{1}$ and whose out-degree vector is $m_{2}$ ?

Complexity: The problem is NP-complete since it contains as a special case $\left(E_{1}=\emptyset\right)$ the NP-complete problem of [19].

\subsection{Mixed $m$-orientation}

MiXed $m$-ORIEnTATION SANDWICH PRoblem

Instance: Given mixed graphs $G_{1}=\left(V, E_{1} \cup A_{1}\right)$ and $G_{2}=$ $\left(V, E_{2} \cup A_{2}\right)$ with $E_{1} \subseteq E_{2}, A_{1} \subseteq A_{2}$ and an non-negative integer vector $m$ on $V$.

Question: Does there exist a sandwich mixed graph $G=$ ( $V, E \cup A$ ) with $E_{1} \subseteq E \subseteq E_{2}$ and $A_{1} \subseteq A \subseteq A_{2}$ that has an orientation $\vec{G}=(V, \vec{E} \cup A)$ whose in-degree vector is $m$ ?

Characterization: Suppose that $E_{1} \subseteq E \subseteq E_{2}$ has been chosen and oriented, then the problem is reduced to the DIReCTEd Degree Constrained SANDWich Problem with $m_{1}(v)=m(v)-d_{\vec{E}}^{-}(v)$ which, by Theorem 8 , has a solution if and only if $d_{A_{2}}^{-}(v) \geq m(v)-d_{\vec{E}}^{-}(v) \geq d_{A_{1}}^{-}(v)$ for all $v \in V$. Hence the MIXED $m$-ORIENTATION SANDWICH PROBLEM has a solution if and only if there exists $E_{1} \subseteq E \subseteq E_{2}$ which admits an orientation $\vec{E}$ with 
$m(v)-d_{A_{1}}^{-}(v) \geq d_{\vec{E}}^{-}(v) \geq m(v)-d_{A_{2}}^{-}(v)$ for all $v \in V$. Let $m_{2}: V \rightarrow \mathbb{Z}$ satisfy $m(v)-d_{A_{2}}^{-}(v) \leq m_{2}(v) \leq m(v)-$ $d_{A_{1}}^{-}(v)$. By Theorem 11, there exists $E_{1} \subseteq E \subseteq E_{2}$ which admits an orientation $\vec{E}$ with $d_{\vec{E}}^{-}(v)=m_{2}(v)$ if and only if $i_{E_{1}}(X) \leq m_{2}(X) \leq e_{E_{2}}(X)$ for all $X \subseteq V$. Therefore we have

Claim 3 The Mixed $m$-ORIEntation SANDwich ProbLEM has a YES answer if and only if there exists an integervalued function $m_{2}: V \rightarrow \mathbb{Z}$ such that, $\forall v \in V$ and $\forall X \subseteq V$,

$m(v)-d_{A_{2}}^{-}(v) \leq m_{2}(v) \leq m(v)-d_{A_{1}}^{-}(v)$,

$i_{E_{1}}(X) \leq m_{2}(X) \leq e_{E_{2}}(X)$.

Claim 4 The pair $\left(i_{E_{1}}, e_{E_{2}}\right)$ is a strong pair.

Proof Let $X, Y, Z$ be three pairwise disjoint subset of $V$. We show that $e_{E_{2}}(X \cup Z)-i_{E_{1}}(Y \cup Z) \geq e_{E_{2}}(X)-i_{E_{1}}(Y)$. In fact, we have $i_{E_{1}}(Y \cup Z)-i_{E_{1}}(Y)=i_{E_{1}}(Z)+d_{E_{1}}(Y, Z) \leq$ $i_{E_{2}}(Z)+d_{E_{2}}(Y, Z)$, and $e_{E_{2}}(X \cup Z)-e_{E_{2}}(X)=i_{E_{2}}(Z)+$ $d_{E_{2}}(Z)-d_{E_{2}}(X, Z)$. As $X, Y, Z$ are pairwise disjoint, $d_{E_{2}}(Y, Z)+d_{E_{2}}(X, Z) \leq d_{E_{2}}(Z)$. The claim follows by Claim 1.

By Claim 3, 4 and Theorem 6 applied for $\alpha(v)=m(v)-$ $d_{A_{2}}^{-}(v), \beta(v)=m(v)-d_{A_{1}}^{-}(v), p=i_{E_{1}}, b=e_{E_{2}}$, we have

Theorem 12 The MIXED $m$-ORIENTATION SANDWICH PROBLEM has a YES answer if and only if

$i_{E_{1}}(X)+\hat{d}_{A_{1}}^{-}(X) \leq m(X) \leq e_{E_{2}}(X)+\hat{d}_{A_{2}}^{-}(X)$

for every subset $X$ of $V$.

Note that Theorem 12 implies Theorems 8 and 11.

Complexity: The condition (15) can be verified in polynomial time by Theorem 4. If it is satisfied, then a vector $m_{2}$ satisfying the conditions in Claim 3 can be found using a greedy algorithm for g-polymatroids. Then we find and orient an edge set $E\left(E_{1} \subseteq E \subseteq E_{2}\right)$ with in-degree $m_{2}$ ( $m$-ORIENTATION SANDWICH PROBLEM). Last, we choose an arc set $A\left(A_{1} \subseteq A \subseteq A_{2}\right)$ such that $d_{A}^{-}(v)=m_{1}(v)=$ $m(v)-m_{2}(v)$, for all $v \in V$ (Directed Degree ConSTRAINED SANDWICH PROBLEM).

\section{Contracting sandwich problems}

In this section, we propose to consider a new type of sandwich problem. Instead of deleting edges from the optional graph, we are interested in contracting edges. We solve the problem for the property $\Pi$ being a bipartite graph.
Contracting SANDwich Problem

Instance: Given an undirected graph $G=(V, E)$ and $E_{0} \subseteq E$.

Question: Does there exist $F \subseteq E_{0}$ such that contracting $F$ results in a bipartite graph?

Complexity: Since a graph is bipartite if and only if all its cycles have an even length, the problem is equivalent to finding $F \subseteq E_{0}$ such that, for all cycles $C,|C \cap F| \equiv|C| \bmod 2$.

Fix a spanning forest $T$ of $G$. For $e \in E \backslash T$, denote $C(T, e)$ the unique cycle contained in $T \cup e$. By [18, Theorem 9.1.2], if $C$ is a cycle of $G$ then $C=\Delta_{e \in C} C(T, e)$, where $\Delta$ denotes the symmetric difference of sets. Therefore, $|C \cap F| \equiv \sum_{e \in C}|C(T, e) \cap F| \bmod 2$. Let $\mathcal{C}_{T}$ denote the collection of cycles $C(T, e)$ of $G$. The problem is reduced to finding $F \subseteq E_{0}$ such that, for all $C \in \mathcal{C}_{T}$, $|C \cap F| \equiv|C| \bmod 2$, or equivalently, finding an $F^{\prime}$ (= $E \backslash F) \supseteq E_{1}=E \backslash E_{0}$ such that $\left|F^{\prime} \cap C\right| \equiv 0 \bmod 2$, for all $C \in \mathcal{C}_{T}$.

Consider now the matrix $M$ defined as the following. The rows of $M$ correspond to $C \in \mathcal{C}_{T}$ and the columns correspond to the edges of $G$; the entry $M_{C e}$ is 1 if $e \in C$ and is 0 otherwise. For $X \subseteq E$, let $\chi_{X}$ denote the characteristic vector of $X$. For a vector $x \in\{0,1\}^{E}$, let $x_{\mid X}$ denote the projection of $x$ on $X$. Let 1 be the all-one vector in $\{0,1\}^{E}$. A subset $F^{\prime} \subseteq E$ satisfies $\left|F^{\prime} \cap C\right| \equiv 0 \bmod 2$, for all $C \in \mathcal{C}_{T}$, if and only if $\chi_{F^{\prime}} \in \operatorname{Ker} M$ in $\mathbb{F}_{2}$. Such an $F^{\prime}$ is the solution of the Contracting Sandwich Problem if and only if $\chi_{F^{\prime} \mid E_{1}}=\mathbf{1}_{\mid E_{1}}$.

Let $B$ be a basis of the kernel of $M$ in $\mathbb{F}_{2}$. (This can be computed in polynomial time using the Gauss elimination.) Consider the projections $B^{\prime}$ of $B$ on $E_{1}$. Then the CONTRACTING SANDWICH PROBLEM has a solution if and only if $\mathbf{1}_{\mid E_{1}}$ is in the subspace of $\{0,1\}^{E_{1}}$ spanned by $B^{\prime}$, that is, $\operatorname{rank} B^{\prime}=\operatorname{rank} B^{\prime} \cup \mathbf{1}_{\mid E_{1}}$. This can be decided in polynomial time using the Gauss elimination. We conclude that the Contracting Sandwich Problem is in P.

We finish with a related problem. For a fixed integer $k$, solving the CONTRACTING SANDWich PROBLEM when $E_{0}=E$ with extra requirement $|F| \leq k$ is known to be tractable in polynomial time [14]. However, the authors mention that finding a solution of minimum cardinality is NP-complete.

Acknowledgements This research was partially supported by CNPq, CAPES (Brazil)/COFECUB (France), FAPERJ.

\section{References}

1. Dantas S, Klein S, Mello CP, Morgana A (2009) The graph sandwich problem for $P_{4}$-sparse graphs. Discrete Math 309:36643673

2. Dantas S, Figueiredo CMH, Golumbic MC, Klein S, Maffray F (2011) The chain graph sandwich problem. Ann Oper Res 188:133-139 
3. Dourado M, Petito P, Teixeira RB, Figueiredo CMH (2008) Helly property, clique graphs, complementary graph classes, and sandwich problems. J Braz Comput Soc 14:45-52

4. Edmonds J (1977) Matroid intersection. Discrete Optim 4:3949 (Proc Adv Res Inst Discrete Optimization and Systems Appl, Banff, Alta)

5. Edmonds J, EL Johnson (1973) Matching, Euler tours and the Chinese postman. Math Program 5:88-124

6. Even S, Itai A, Shamir A (1976) On the complexity of timetable and multicommodity flow problems. SIAM J Comput 5:691-703

7. Figueiredo $\mathrm{CMH}$, Faria L, Klein S, Sritharan R (2007) On the complexity of the sandwich problems for strongly chordal graphs and chordal bipartite graphs. Theor Comput Sci 38:57-67

8. Ford LR, Fulkerson DR (1962) Flows in Networks. Princeton University Press, Princeton

9. Frank A (1982) An algorithm for submodular functions on graphs. Ann Discrete Math 16:97-120

10. Frank A, Gyárfás A (1976) How to orient the edges of a graph. Colloq Math Soc János Bolyai 18:353-364

11. Frank A, Tardos E (1988) Generalized polymatroids and submodular flows. Submodular optimization. Math Program 42(3):489563

12. Golumbic MC, Kaplan H, Shamir R (1995) Graph sandwich problems. J Algorithms 19(3):449-473

13. Hakimi SL (1965) On the degrees of the vertices of a directed graph. J Franklin Inst 279(4):290-308

14. Heggernes P, van't Hof P, Lokshtanov D, Paul C (2011) Obtaining a bipartite graph by contracting few edges. In: Proceedings of
IARCS annual conference on foundations of software technology and theoretical computer science, pp 217-228

15. Hoffman AJ (1960) Some recent applications of the theory of linear inequalities to extremal combinatorial analysis. Proc Symp Appl Math 10:113-127

16. Holyer I (1981) The NP-completeness of edge-coloring. SIAM J Comput 10(4):718-720

17. Iwata S, Fleischer L, Fujishige S (2001) A combinatorial strongly polynomial algorithm for minimizing submodular functions. J ACM 48(4):761-777

18. Oxley JG (1992) Matroid theory. Oxford University Press, New York

19. Pálvölgyi D (2009) Deciding soccer scores and partial orientations of graphs. Acta Univ Sapientiae, Math 1(1):35-42

20. Schrijver A (2003) Combinatorial optimization: polyhedra and efficiency. Algorithms and combinatorics, vol 24. Springer, Berlin

21. Schrijver A (2000) A combinatorial algorithm minimizing submodular functions in strongly polynomial time. J Comb Theory, Ser B 80(2):346-355

22. Sritharan R (2008) Chordal bipartite completion of colored graphs. Discrete Math 308:2581-2588

23. Tardos E (1985) A strongly polynomial minimum cost circulation algorithm. Combinatorica 5(3):247-255

24. Teixeira RB, Dantas S, Figueiredo CMH (2010) The polynomial dichotomy for three nonempty part sandwich problems. Discrete Appl Math 158:1286-1304

25. Tutte WT (1952) The factors of graphs. Can J Math 4:314-328 